\title{
Correction to: Hispanic Spinocerebellar Ataxia Type 35 (SCA35) with a Novel Frameshift Mutation
}

\section{Chih-Chun Lin ${ }^{1} \cdot$ Shi-Rui Gan ${ }^{2} \cdot$ Deepak Gupta $^{3} \cdot$ Armin Alaedini $^{4,5} \cdot$ Peter H Green ${ }^{4,5} \cdot$ Sheng-Han Kuo ${ }^{3}$}

Published online: 7 August 2020

(C) Springer Science+Business Media, LLC, part of Springer Nature 2020

\section{Correction to: The Cerebellum (2019) 18:291-294.} https://doi.org/10.1007/s12311-018-0978-6

The authors found out that there was a mistake in the Table 2 of the published paper.

The mutation listed in the last row of this table should be c.841delC instead of c.841delG.

This error does not have any effect on the results and findings of this paper.

Publisher's Note Springer Nature remains neutral with regard to jurisdictional claims in published maps and institutional affiliations.

The online version of the original article can be found at https://doi.org/ 10.1007/s12311-018-0978-6

\section{Sheng-Han Kuo}

sk3295@columbia.edu

1 Methodist Neurological Institute, Houston, TX, USA

2 Department of Neurology and Institute of Neurology, The First Affiliated Hospital of Fujian Medical University, Fujian, China

3 Department of Neurology, Columbia UniversityMedical Center, 650 West 168th Street, Room 305, New York, NY 10032, USA

4 Department of Medicine, Columbia University Medical Center, New York, NY, USA

5 Celiac Disease Center, College of Physicians and Surgeons, Columbia University, New York, NY, USA 